\title{
Diagnósticos de enfermagem de prematuros sob cuidados intermediários
}

\author{
Nursing diagnoses of premature infant under intermediary care \\ Diagnósticos de enfermería de prematuros bajo la atención intermediária
}

\begin{abstract}
Natália Del'Angelo', Fernanda dos Santos Nogueira de Góes', Maria Célia Barcellos Dalri', Adriana Moraes Leite', Maria Cândida de Carvalho Furtado', Carmen Gracinda Silvan Scochi'

'Universidade de São Paulo. Escola de Enfermagem de Ribeirão Preto. Programa de Pós-graduação Enfermagem. Ribeirão Prero, $S P$
\end{abstract}

Submissão: 27/07/2009

Aprovação: $31 / 07 / 2010$

\section{RESUMO}

O estudo teve como objetivo identificar diagnósticos de enfermagem de prematuros assistidos em uma unidade de cuidado intermediário neonatal do interior de São Paulo, Brasil. Tratou-se de um estudo retrospectivo a partir de prontuários de 118 prematuros hospitalizados. Os diagnósticos de enfermagem mais freeuentes foram: Privação do Sono (83, 1\%), Risco de Infecção (76,3\%) e Processos Familiares Alterados $(75,4 \%)$, pertencentes aos respectivos domínios da NANDA: atividade/repouso, segurança/proteção e relacionamentos de papel. Os resultados do estudo articularam a freeuência dos diagnósticos com os domínios da NANDA aos Quais pertencem, identificando limitação da abordagem dos mesmos e principais domínios apontados na sistematização da assistência ao recém-nascido prematuro em cuidado intermediário.

Descritores: Diagnóstico de enfermagem; Prematuro; Terapia Intensiva Neonatal, Enfermagem neonatal.

\section{ABSTRACT}

The study aims at identifying nursing diagnoses of premature infants attended in a neonatal intermediary care unit in the countryside of São Paulo State, Brazil. That was a retrospective study conducted from 118 patient records of hospitalized premature infants. The most frequent nursing diagnosis were sleep deprivation (83.1\%), risk for infection (76.3\%) and dysfunctional family processes $(75.4 \%)$, belonging to NANDA domains: activity/rest, security/protection and role relations, this order. Study results articulated the diagnosis frequency with NANDA domain to which they belong identifying their approach limitation and main domains pointed in assistance systematization to premature newborn in intermediary care.

Key words: Nursing diagnosis; Infant, premature; Intensive care, neonatal; Neonatal nursing.

\section{RESUMEN}

El estudio tuvo como objetivo identificar los diagnósticos de enfermería de prematuros asistidos en una unidad de cuidado intermediario neonatal del interior de Sao Paulo, Brasil. Re realizó un estudio retrospectivo a partir de los registros hospitalarios de prematuros hospitalizados. Los diagnósticos más frecuentes fueron: Perturbación del Sueño (83, 1\%), Riesgo de Infección (76,3\%) y Alteración de los Procesos Familiares $(75,4 \%)$, pertenecientes a los dominios NANDA: actividad/reposo, seguridad/protección y papel/relación, en esta orden. Los resultados del estudio articularon la frecuencia de los diagnósticos con los dominios NANDA pertenecientes, identificando limitación del abordaje de los mismos y principales dominios apuntados en la sistematización de la asistencia al recién-nascido prematuro en cuidado intermediario.

Descriptores: Diagnóstico de Enfermería; Prematuro; Cuidado intensino neonatal; Enfermería neonatal. 


\section{INTRODUÇÃO}

A Sistematização da Assistência de Enfermagem (SAE) é definida pela maioria dos enfermeiros brasileiros como sendo a organização assistencial direcionada ao paciente, família ou comunidade, porém há uma Questão semântica importante a se considerar visto Que sistematizar pode ser simplesmente o ato de organizar sem Que sejam utilizadas todas as etapas do método científico - denominado Processo de Enfermagem ${ }^{(1)}$.

A resolução 358-2009 do Conselho Federal de Enfermagem $\operatorname{COFEN}^{(2)}$, ao resolver sobre a realização da SAE, considera Que a mesma organiza o trabalho do profissional frente ao método, sua equipe e instrumentos operacionalizando o processo de enfermagem. Na realidade assistencial, algumas medidas vêm sendo elaboradas no sentido de auxiliar o planejamento, execução e avaliação dos cuidados de enfermagem. Tais medidas referem-se à implementação do processo de enfermagem.

Para Que seja efetiva tal implementação são necessários ao enfermeiro habilidade interpessoal, raciocínio e elaboração de ideias, valores e crenças; características Que subsidiam a prática do método de acordo com os preceitos do mesmo ${ }^{(3)}$.

O processo de enfermagem pode ser considerado sistemático, por consistir em etapas com iniciativas deliberadas para maximizar a eficiência e obter resultados em longo prazo; dinâmico, por proporcionar idas e vindas para formulação de diagnósticos e, humanizado, por atender as necessidades de corpo, mente e espírito de forma individualizada ${ }^{(4)}$. Estudo ${ }^{(5)}$ afirma Que a prática do processo de enfermagem é influenciada pela concepção de cuidado adotada pelo enfermeiro, havendo, portanto, diversas abordagens e formas de cuidar estruturadas sobre diferentes teorias e modelos conceituais.

A divulgação deste método no Brasil, iniciada na década de 70 por Horta, apresenta implicações até a atualidade tendo aplicação de impacto na assistência, ensino e pesQuisa ${ }^{(6-7)}$. Uma das fases do processo de enfermagem Que merece destaque devido sua complexidade de elaboração é o Diagnóstico de Enfermagem (DE), havendo diferentes concepções a depender da abordagem teórica utilizada. No presente estudo adotamos o referencial da North American Nursing Diagnosis Association (NANDA) - Que define o DE como um "julgamento clínico sobre as respostas do indivíduo, da família ou da comunidade a problemas de saúde / processos vitais reais ou potenciais" e "é a base para selecionar as intervenções de enfermagem com foco nos resultados que se espera obter"(8).

A tomada de decisões em enfermagem se norteia pelo julgamento clínico elaborado pelo enfermeiro através de embasamento científico e auxiliado pela experiência, percepção e intuição na busca por elaborar julgamentos baseados em evidências. Tal processo desencadeia o diagnóstico de enfermagem ${ }^{(9)}$.

A utilização do DE na assistência de enfermagem é importante para a individualização do cuidado e subsidia a execução e a avaliação da assistência com base em um raciocínio clínico registrado de forma organizada. Sua vinculação à prática clínica oferece ao enfermeiro possibilidade de diagnosticar situações de sua responsabilidade (a respeito da saúde do paciente) e, assim, controlar as mudanças de estado ${ }^{(10)}$. Embora constitua um elemento fundamental da prática em enfermagem, observa-se a escassez de peseuisas sobre essa temática na área de Enfermagem Neonatal.
Nessa área há um ramo de estudos focado na prematuridade enQuanto situação peculiar no processo de saúde do neonato. $\mathrm{O}$ prematuro demanda cuidados diferenciados e acompanhamento específico para suas necessidades, tendo-se preocupação especial com os DE desse segmento populacional durante a hospitalização, Quer seja em unidade de terapia intensiva neonatal (UTIN), com alta complexidade de cuidado e peculiar aos neonatos de alto risco com instabilidade clínica e/ou gravemente enfermos, ou em unidade de cuidado intermediário neonatal (UCIN), Que atende a uma demanda de neonatos de médio risco com estabilidade clínica, mas Que necessitam de observação e de algumas tecnologias de cuidado de menor complexidade.

$\mathrm{Na}$ literatura nacional e internacional, foram encontrados 21 estudos relacionados aos DE em Neonatologia (incluindo pré-termo e a termo) com temas como: aleitamento materno ${ }^{(11-12)}$, cardiopatia congênita $^{(13-14)}$, dor ${ }^{(15)}$, pais/família ${ }^{(16-17)}$, visão do aluno a respeito do $\mathrm{DE}^{(18)}$, doenças respiratórias ${ }^{(19)}$, hipotermia ${ }^{(20)} \mathrm{e}$ a elaboração de guia de intervenção para os DE aplicado aos neonatos em UTIN(21). Destes estudos apenas Quatro ${ }^{(14-15,20-21)}$ não especificaram o referencial teórico de diagnósticos de enfermagem utilizados, sendo Que os demais utilizaram a NANDA.

Constata-se aí a escassez de pesquisas na temática do DE relacionado ao cuidado do prematuro em unidades neonatais, motivando a realização deste estudo com o objetivo de identificar esses diagnósticos aplicados aos prematuros assistidos em uma UCIN do interior de São Paulo, Brasil.

Espera-se com isto trazer subsídios para a organização dessa assistência e contribuir com reflexões Que instrumentalizam a formação e educação permanente dos enfermeiros com vistas à elaboração dos DE.

\section{METODOLOGIA}

Tratou-se de estudo retrospectivo, o Qual consistiu em descrever situações, características ou propriedades, ou ainda relacioná-las a um determinado fenômeno(21).

Os dados foram obtidos a partir de um levantamento de prontuários dos prematuros assistidos na UCIN de um hospital universitário do interior paulista, Que é de referência terciária na atenção perinatal. A equipe de enfermagem dessa unidade é constituída por seis enfermeiros e 26 auxiliares de enfermagem, distribuídos em três turnos de trabalhos para atendimento aos neonatos distribuídos em Quatro enfermarias.

Nessa unidade neonatal, o processo de enfermagem foi implantado em 2000 e revisado por duas vezes em anos subsequentes. Para a operacionalização do processo são utilizados impressos Que subsidiam o cumprimento de cada uma das etapas, a saber: (1) coleta de dados - histórico de enfermagem e exame físico diário norteado por necessidades humanas básicas, conforme referencial de Horta, formulário de admissão na UCIN contendo dados do neonato e da mãe, gráfico de sinais vitais e dados antropométricos; (2) diagnósticos de enfermagem; (3) evolução e (4) prescrição de enfermagem; (5) avaliação - planejamento da alta (condições da habitação, orientações e cuidados demonstrados e vacinação) e orientações na alta hospitalar.

O processo de enfermagem é desenvolvido rotineiramente, sendo o exame físico, os diagnósticos e as prescrições de enfermagem 
realizadas a cada 24 horas, ou antes, se necessário, mediante o preenchimento de formulários em check list; a evolução de enfermagem é realizada de forma discursiva, a cada turno de trabalho (manhã, tarde e noite).

No check list relativo aos DE constam 2 I diagnósticos neonatais, adaptados da NANDA e há espaço livre para incorporação de outros diagnósticos não ali contidos. Destaca-se Que os DE elencados não contemplam todos os domínios e nem são sistematicamente revisados.

Para a coleta dos dados foi elaborado um instrumento específico no Qual foram registrados os dados relativos ao nascimento, à internação e aos DE. Para o registro dos DE, foram incluídos no instrumento todos os diagnósticos já preestabelecidos na UCIN contidos no impresso específico utilizado diariamente pelos enfermeiros. Durante a coleta de dados, uma vez presente o DE no prontuário do RN, esse era registrado apenas uma vez, independente do momento e da frequência de aparecimento durante todo o processo de hospitalização. Foram coletados apenas os títulos dos DE, cujos enunciados foram adaptados segundo a NANDA 2007/2008 ${ }^{(8)}$. O período de internação dos prematuros incluídos na pesquisa foi de 01 de janeiro a 31 de julho de 2007. envolvendo $118 \mathrm{RN}$.

A pesquisa foi aprovada pelo Comitê de Ética em Pesquisa do hospital, com dispensa do termo de consentimento pós-informado. Processo $n^{\circ}$ 5229/2008.

\section{RESULTADOS E DISCUSSÃO}

Dos 118 prematuros predominaram indivíduos do sexo masculino (61\%), nascidos de parto cesáreo $(60,2 \%)$ e gestação única (78\%), com idade gestacional (IG) entre 32 e 36 semanas $(60,2 \%)$ e peso ao nascer entre $2000 \mathrm{~g}$ e $2499 \mathrm{~g}$.

No levantamento dos prontuários constatou-se Que 74,6\% prematuros não continham formulário de DE preenchido no dia da admissão e $54,1 \%$ no dia da alta hospitalar.

A Tabela I apresenta os DE registrados nos prontuários dos prematuros incluídos no estudo.

Dentre os 21 DE contidos no impresso próprio da enfermagem, os mais frequentes foram: privação do sono $(83,1 \%)$, risco de infecção $(76,3 \%)$ e processos familiares alterados $(75,4 \%)$.

PesQuisa $^{(22)}$ realizada com mãe e gemelares prematuros também identificou DE semelhantes aos encontrados neste estudo e Que são pertinentes ao processo de internação em UCIN, são eles: dificuldade de sucção e deglutição, integridade da pele compro-metida, mucosas oral e genital comprometidas, conhecimento deficiente da genitora para cuidar das filhas e vínculo mãe-filho prejudicado. Ressaltase, entretanto que a pesquisa utilizou a Classificação Internacional para a Prática de Enfermagem (CIPE 1.0).

Muitos DE são decorrentes de problemas apresentados pelos prematuros em unidades neonatais como imaturidade fisiológica, riscos de injúrias reversíveis ou irreversíveis decorrentes do processo de internação em UTIN, imaturidade neurológica e sensorial para modulação dos agentes estressores do ambiente e interrupção do sistema familiar. As intervenções devem ser realizadas para Que os seguintes resultados sejam alcançados: estabilidade fisiológica e crescimento, injúria estrutural mínima, competência neurocomportamental estabilidade e integração familiar ${ }^{(23)}$.

Apesar do formulário da UCIN conter a listagem de $21 \mathrm{DE}$, seis deles não foram registrados nos prontuários dos prematuros analisados: Perfusão Tissular Alterada, Diarreia, Resposta Disfuncional ao Desmame Ventilatório, Troca de Gases Prejudicada, Excesso de Volume de Líquidos e Déficit de Volume de Líquidos.

Questiona-se a ausência de alguns destes DE, como por exemplo a Troca de Gases Prejudicada, pois muitos dos prematuros extremos em UCIN apresentam broncodisplasia pulmonar e permanecem dependentes de oxigênio por longos períodos. Acredita-se Que, possivelmente, os enfermeiros tenham substituído tal diagnóstico por padrão respiratório ineficaz e/ou ventilação espontânea prejudicada, embora tenham sido registrados em frequência reduzida, $16,1 \%$ e $2,5 \%$ respectivamente.

Outra limitação do sistema de registro refere-se à ausência de I I DE da NANDA ${ }^{(8)}$ no check list, os Quais têm a prematuridade como fator relacionado ou de risco, a saber: Amamentação Ineficaz, Disposição para Aumento da Competência Organizacional do Bebê, Comportamento Desorganizado do Bebê, Risco de Comportamento Desorganizado do Bebê, Risco de Crescimento Desproporcional, Deglutição Prejudicada, Risco de Atraso no Desenvolvimento, Risco de Síndrome da Morte Súbita do Bebê, Paternidade ou Maternidade Prejudicada, Risco de Paternidade ou Maternidade Prejudicada e Risco de Volume de Líuuidos Deficiente.

Assim, os DE encontrados podem não refletir a real necessidade

Tabela 1. Distribuição dos DE dos 118 prematuros assistidos na UCIN do hospital universitário do interior paulista, no período de janeiro a julho 2007.

\begin{tabular}{lc}
\hline DIAGNÓSTICOS DE ENFERMAGEM & $\%$ \\
\hline Privação do sono & 83,1 \\
Risco de infecção & 76,3 \\
Processos familiares interrompidos & 75,4 \\
Dor & 65,3 \\
Risco de integridade da pele prejudicada & 61,0 \\
Atraso no crescimento e no desenvolvimento & 52,5 \\
Risco de aspiração & 46,6 \\
Amamentação interrompida & 45,8 \\
Termorregulação ineficaz & 44,1 \\
Mucosa oral prejudicada & 42,4 \\
Risco de vínculo pais/illho alterado & 39,0 \\
Risco de deseQuilíbrio na temperatura & 35,6 \\
Corporal & \\
Padrão respiratório ineficaz & 16,1 \\
Padrão ineficaz de alimentação do bebê & 6,8 \\
Ventilação espontânea prejudicada & 2,5 \\
Proteção ineficaz & 1,7 \\
Capacidade adaptativa diminuída intracraniana & 1,7 \\
Integridade da pele prejudicada & 0,8 \\
Desobstrução ineficaz de vias aéreas & 0,8 \\
Constipação & 0,8 \\
Nutrição deseQuilibrada & 0,8 \\
\hline
\end{tabular}


Tabela 2. Distribuição dos domínios da NANDA e respectivos DE registrados para os 118 prematuros do estudo, no período de janeiro a julho de 2007.

\begin{tabular}{lc}
\hline DOMÍNIOS DA NANDA (DE) & $\%$ \\
\hline $\begin{array}{l}\text { Segurança/proteção } \\
\text { (risco de infecção, risco de integridade da pele prejudicada, risco de aspiração, }\end{array}$ \\
$\begin{array}{l}\text { termorregulação ineficaz, mucosa oral prejudicada, risco de desequilibrio na temperatura } \\
\text { corporal, proteção ineficaz, integridade da pele prejudicada e desobstrução ineficaz das vias } \\
\text { aéreas) }\end{array}$ & 42,8 \\
$\begin{array}{l}\text { Atividade/repouso } \\
\text { (privação do sono, padrão respiratório ineficaz e ventilação espontânea prejudicada) }\end{array}$ & 14,3 \\
$\begin{array}{l}\text { Relacionamentos de papel } \\
\text { (processos familiares interrompidos, amamentação interrompida e risco de vínculo pais/filho }\end{array}$ & 14,3 \\
alterado) & \\
$\begin{array}{l}\text { Nutrição } \\
\text { (padrão ineficaz de alimentação do bebê e nutrição deseQuilibrada) }\end{array}$ & 9,5 \\
$\begin{array}{l}\text { Eliminação/troca } \\
\text { (constipação) }\end{array}$ & 4,7 \\
$\begin{array}{l}\text { Enfrentamento/tolerância ao estresse } \\
\text { (capacidade adaptativa diminuída intracraniana) }\end{array}$ & 4,7 \\
$\begin{array}{l}\text { Conforto } \\
\text { (dor) }\end{array}$ & \\
$\begin{array}{l}\text { Crescimento/desenvolvimento } \\
\text { (atraso no crescimento e no desenvolvimento) }\end{array}$ & 4,7 \\
\hline
\end{tabular}

grande pertinência, mas considera-se que a Quase totalidade deles deveria apresentar esse $\mathrm{DE}$, pois além de consonante com a ideia de Que todos os neonatos estão sujeitos à infecção devido à transição do meio estéril (útero materno) para ambiente contaminado, há ainda a fragilidade biológica do prematuro com imaturidade de diversos sistemas, em especial o imunológico, acrescida da utilização de procedimentos de alta complexidade de apoio ao diagnóstico e à terapêutica e do longo período de internação, tornando, portanto, essas crianças mais suscetíveis a infecções e ao adoecimento ${ }^{(24)}$.

Acredita-se Que poderiam dispensar tal DE aQueles prematuros Que apresentam infecção, portanto com DE Proteção Ineficaz, caracterizando uma condição clínica com maior vulnerabilidade a outras infecções.

Assim, o DE proteção ine-

dos prematuros em UCIN pelo fato de se ter utilizado fonte secundária e o prontuário constituir-se em fonte de dados limitada. Aspecto evidenciado no presente estudo, pois conforme apontado, o sistema de registro disponível na UCIN não é frequentemente revisado e, apesar de haver espaço livre no check list para a inclusão de outros DE não elencados, não se encontrou a inclusão de QualQuer outro DE nos prontuários.

Neste aspecto, considera-se Que instrumento do tipo check list tanto pode contribuir com a praticidade de documentação da SAE como pode desestimular o profissional a incluir outros dados para além do Que está listado, no caso os DE.

Dentre os 13 domínios da NANDA ${ }^{(8)}$, cinco deles não foram localizados entre os DE presentes no instrumento da unidade e também não foram localizados nos prontuários analisados (promoção da saúde, percepção/cognição, autopercepção, sexualidade e princípios de vida).

A Tabela 2 indica a frequência de aparecimento dos domínios da NANDA segundo os DE registrados pelos enfermeiros da UCIN.

O domínio registrado com maior frequência é denominado Segurança/Proteção $(42,8 \%)$ e indica "estar livre de perigo, lesão física ou dano do sistema imunológico, preservação contra perdas, proteção da segurança e seguridade"(8). Podemos afirmar Que a freQuência encontrada reflete algumas das necessidades de saúde mais comuns na população estudada e também as intervenções da equipe assistencial na UCIN no âmbito das necessidades básicas primordiais do prematuro: controle da temperatura corporal, sistema imunológico para regular os processos celulares, ventilação adequada e a proteção da pele e mucosas. Dentro deste domínio, o DE Risco de Infecção foi freeuente entre os prematuros indicando ficaz é mais amplo e inclui tanto os cuidados necessários para a prevenção como para o tratamento de infecção e, portanto, tornase dispensável registrar os dois diagnósticos.

A presença ou o Risco de Integridade da Pele Prejudicada também estão subestimados nos registros dos enfermeiros, em especial o primeiro DE Que foi registrado em frequência muito baixa $(0,8 \%)$, pois os prematuros apresentam diversos fatores relacionados como extremos de idade, além da presença de fatores mecânicos como adesivos para fixação de cateteres e sondas, uso de fraldas e as proeminências ósseas. Cabe assinalar que a pele do recém-nascido pré-termo é delicada e propensa a lesões, sendo fundamental que a equipe de enfermagem esteja sempre atenta aos cuidados diários com o objetivo de minimizar comprometimento da integridade da pele.

A identificação do DE Mucosa Oral Prejudicada reflete os cuidados necessários à higiene bucal de pacientes internados em unidades críticas, pois é essencial para evitar a proliferação de bactérias e fungos, Que, além de prejudicar a saúde bucal e o bemestar do paciente pode propiciar outras infecções e doenças sistêmicas. As infecções estão entre as principais causas de morbidade e mortalidade entre pacientes internados em unidades de cuidados intensivos ${ }^{(25)}$.

Ainda sobre este domínio, considerando sua alta frequência dentre os demais, deve-se refletir sobre a sondagem gástrica enQuanto um procedimento imprescindível no auxílio à nutrição de prematuros, com até 32 semanas de idade gestacional, aproximadamente, pois os mesmos ainda não conseguem coordenar os reflexos de sucção e de deglutição ${ }^{(26)}$. Desta forma, considerase Que Risco de Aspiração é um diagnóstico pertinente a este 
segmento populacional, uma vez Que possibilita a elaboração de plano assistencial adequado aos RN.

Tais limitações fisiológicas de muitos prematuros, acrescidos dos frequentes problemas respiratórios apresentados, da necessidade de nutrição parenteral, gástrica e entérica, dentre outros aspectos, justificam os DE Padrão Ineficaz de Alimentação do Bebê e Nutrição Desequilibrada, incluídos no domínio Nutrição $(9,5 \%)$, todavia as baixas frequências encontradas também sugerem o subregistro de enfermagem.

Com base nos domínios Atividade/Repouso (14,3\%) definido como "produção, conservação, gasto ou equilíbrio de recursos energéticos ${ }^{*(8)}$ e Crescimento/Desenvolvimento (4,7\%) denominado "aumentos apropriados para a idade nas dimensões físicas, maturação de sistemas orgânicos e/ou progressão através dos estágios de desenvolvimento "(8), devemos salientar a importância do cuidado desenvolvimental. A superestimulação ambiental, com ruídos intensos, iluminação contínua, manipulação excessiva, exposição a procedimentos dolorosos e interrupções repetidas dos ciclos de sono do prematuro, pode repercutir na maturação física e neurológica e na Qualidade de vida destas crianças. A reatividade dos prematuros ao excesso de estímulo pode ser manifestada por alterações nos seguintes sistemas: autônomo - manifestado nas funções fisiológicas; motor - observado pela postura, tônus muscular e movimentos corporais; e de informação - observado através dos estados de consciência ${ }^{(15-16)}$.

A Dor Aguda consiste no único DE registrado pela enfermagem da UCIN enquadrado no domínio Conforto (4,7\%). Aponta-se que neonatos expostos repetidamente a estímulos dolorosos acumulam os efeitos nocivos da dor e estresse de internação, podendo resultar em consequências negativas ao desenvolvimento neurológico dessas crianças $^{(15)}$. O fato de esse DE ser um dos cinco mais frequentemente registrados nos prontuários dos prematuros assistidos na UCIN denota a preocupação da enfermagem com o manejo da dor do prematuro, na perspectiva do cuidado desenvolvimental e humanizado.

O domínio Relacionamento de Papel (14,3\%) remete aos cuidados relacionados à necessidade de repouso e também situação familiar e de amamentação no contexto da prematuridade. A Amamentação Interrompida pode demonstrar várias situações como o desinteresse da mãe pelo aleitamento materno, a falta de incentivo, a falta de estrutura ou mesmo a hipogalactia decorrente dos longos períodos de internação.

Estudo $^{(I)}$ realizado com 35 mães de filhos prematuros internados em UTIN demonstrou a ocorrência de 100\% para o DE Risco para Amamentação Ineficaz com os seguintes fatores de risco: prematuridade; oportunidade insuficiente para a amamentação ao seio, devido ao recém-nascido estar hospitalizado; déficit de conhecimento Quanto à manutenção da lactação; medo materno; inconstância da sucção do seio devido à separação; alimentação artificial do neonato. Tais dados corroboram com os de outro estudo $^{(12)} \mathrm{O}$ Qual demonstrou Que o desmame precoce decorre da condição clínica do prematuro, do período prolongado de internação, do estresse materno, da falta de rotinas sistematizadas de incentivo ao aleitamento materno e manutenção da lactação, medo e insegurança além das determinações socioculturais.

Outros DE neste domínio são Processos Familiares Interrompidos e Risco para Vínculo Pais/Filhos Alterado, os Quais decorrem de problemas avaliados pelo enfermeiro como sociais e emocionais na relação entre os pais e o prematuro durante o período de internação. Verifica-se neste estudo elevadas frequências para estes DE (74,5\% e 39\%, respectivamente), atribuídas ao enfrentamento das dificuldades Que os pais apresentam frente à prematuridade do bebê, especificidades no cuidado e longa permanência hospitalar. Em uma UTIN brasileira, 59 enfermeiros validaram o DE Conflito no Desempenho do Papel de Pai/Mãe e identificaram as seguintes características definidoras com escores superiores a 80\%: mãe ou pai expressa sentimento de inadequação para atender as necessidades do filho durante a hospitalização; mãe ou pai expressa sentimento de inadequação para atender as necessidades do filho em casa; mãe ou pai expressa preocupação em relação a mudanças no papel paterno/materno e mãe ou pai expressa preocupação em relação à saúde da família ${ }^{(27)}$.

Outro estudo demonstrou Que entre 240 mães com até 24 anos, 24,3\% apresentavam o diagnóstico Maternidade Prejudicada com os seguintes fatores de risco: mãe ter sofrido violência Quando criança, falta de conhecimento sobre a maternidade e a passagem da adolescência para a vida adulta. Como medidas preventivas para tal diagnóstico o estudo cita a atuação da enfermeira na educação como medida de prevenção da gravidez na adolescência, o prénatal com orientações específicas a este seguimento populacional e o acompanhamento no parto e puerpério ${ }^{(28)}$.

Tais resultados corroboram com os dados encontrados neste estudo, demonstrando a importância dos diagnósticos relacionados à família do prematuro. Uma das ações básicas da enfermagem é apoiar a família durante a internação do prematuro, facilitar a formação do apego entre pais e filho e prepará-los para o cuidado dele. Nessa teia de relações se inserem os fatores de incômodo por não ter o filho no ambiente familiar e não poder, imediatamente após o nascimento, inserí-lo no contexto social em Que a família vive $^{(17,28-29)}$. Acrescenta-se também Que há outros DE possíveis para a situação de maternidade/paternidade e família os Quais não foram incluídos no check list da UCIN, são eles: tensão do papel de cuidador, risco de tensão do papel de cuidador, paternidade ou maternidade prejudicada, risco de paternidade ou maternidade prejudicada, disposição para paternidade ou maternidade melhorada, desempenho de papel ineficaz e conflito no desempenho do papel de pai/mãe.

Quanto aos DE registrados em menores frequências, acreditase Que também estão subestimados, exceto aQueles relativos aos domínios Enfrentamento/tolerância ao estresse (4,7\%) e Eliminação/ troca $(4,7 \%)$, pois os diagnósticos registrados são pertinentes a anomalias pouco incidentes na clientela assistida na UCIN.

A expectativa por maior incidência justifica-se pelas características definidoras ou fatores relacionados estabelecidos na $\operatorname{NANDA}^{(8)}$, os Quais apresentam a prematuridade como fator relacionado ou fator de risco e discutidos anteriormente.

As lacunas apontadas anteriormente podem ser entendidas pelo fato de o conhecimento a respeito dos DE ainda ser muito limitado, uma vez Que as outras fases do processo de enfermagem têm se sobressaído em termos de estudos e desenvolvimento ${ }^{(29)}$. Há também a resistência dos profissionais a esse método por acreditarem Que o seu uso é complexo e de aplicação prática inviável devido a não adequação à realidade, dificuldade de compreensão e operacionalização. Além disso, há fatores inerentes ao cenário de 
ensino-aprendizagem. Neste sentido, estudo realizado com alunos do sexto semestre de enfermagem demonstrou que os alunos consideram o ensino do DE como instrumento básico para a assistência de enfermagem e solicitaram a inclusão do conteúdo no terceiro semestre de graduação; também afirmaram Que o aprendizado do DE trouxe modificações sobre o conceito de assistência de enfermagem ${ }^{(18)}$.

\section{CONSIDERAÇÕES FINAIS}

Apesar das limitações do estudo decorrente do uso de fontes secundárias, com foco no formulário da UCIN contendo check list dos DE, constatou-se Que os principais diagnósticos foram registrados nos prontuários e as considerações sobre suas frequências permitem afirmar Que há uma ampla abordagem das necessidades de saúde dos prematuros na UCIN, mas também há lacunas com sub-registro de alguns outros DE pertinentes a esse segmento populacional.

Os resultados apontam para a necessidade de capacitação e educação permanente dos enfermeiros para elaboração dos DE, enquanto uma etapa importante do processo de enfermagem. Para tal recomenda-se a utilização de estratégias Que permitam a visualização dos processos cognitivos (como o raciocínio diagnóstico) desenvolvidos pelo enfermeiro e uso de modelos de raciocínios hipotéticos (observações, softwares, simulação, jogos, estudos de casos e/ou situações-problema), sendo essas possibilidades para redução destas dificuldades.

Acredita-se também Que a inclusão de recursos informatizados ${ }^{(30)}$ como forma de estimular a aQuisição de conhecimentos seja uma forma de facilitar e democratizar o aprendizado. Neste sentido, o software elaborado com esta finalidade e destinado à semiotécnica e semiologia do prematuro, criado para alunos de graduação e enfermeiros, pode ser utilizado como ferramenta de educação permanente. É necessário também transformar em linguagem virtual conteúdos acerca dos DE para prematuros internados em unidades neonatais, cujo web site está em desenvolvimento pelo grupo de pesquisa e constitui motivação para estudos futuros.

A partir do desenvolvimento desses recursos tecnológicos podese incrementar o aprendizado e a prática de elaboração dos DE, fundamentado em bases teóricas reconhecidas e validadas para a obtenção de um padrão de informações na SAE e favorecer a adesão dos profissionais de saúde.

\section{REFERÊNCIAS}

I. Carvalho EC, Bachion MM, Dalri MCB, Jesus CAC. Obstáculos para a implementação do processo de Enfermagem no Brasil. Rev Enf UFPE On Line 2007; I (1): 95-9.

2. Conselho Federal de Enfermagem (BR). Resolução COFEN 358/ 2009. Dispões sobre Sistematização da Assistência de Enfermagem - SAE. Rio de laneiro: COFEN; 2009.

3. Lunney M. Pensamento crítico e diagnóstico de enfermagem: estudo de casos e análises. Porto Alegre: Artmed; 2004.

4. Alfaro-Lefevre R. Aplicação do processo de enfermagem: promoção do cuidado colaborativo. $5^{\mathrm{a}}$ ed. Porto Alegre: Artmed; 2005.

5. Carvalho EC, Garcia TR. Processo de enfermagem: o raciocínio e julgamento clínico no estabelecimento do diagnóstico de enfermagem. In: Anais do $3^{\circ}$ Fórum Mineiro de Enfermagem; 2002; Uberlândia (MG), Brasil. Uberlândia: UFU; 2002. p. $29-40$.

6. Marques LVP, Carvalho DV. Sistematização da assistência de enfermagem em centro de tratamento intensivo: percepção das enfermeiras. Rev Min Enferm 2005; 9(3): 199-205.

7. Cunha SB, Barros ALB. Análise de implementação da Sistematização da Assistência de Enfermagem, segundo o Modelo Conceitual de Horta. Rev Bras Enferm 2005; 58(5): 568-72.

8. North American Nursing Diagnosis Association. Diagnóstico de Enfermagem da NANDA: definições e classificações - 2007/ 2008. Porto Alegre: Artmed; 2008.

9. Christensen IP. Assessment overview of data collection. In: Christensen JP; Kenney-Griffith JW. Nursing process-application of theories, frameworks and models. $2^{\text {nd }}$ ed. Saint Louis: Mosby; 1986.

10. Carpenito-Moyet LJ. Diagnósticos de enfermagem: aplicação à prática clínica. $10^{\mathrm{a}}$ edição. Porto Alegre: Artmed; 2005.

11. Viera CS. Risk of ineffective breast-feeding: a nursing diagnosis. Rev Bras Enferm 2004; 57(6): 712-4
12. Abrão ACFV, Gutiérrez, MGR, Marin HF. Utilização do diagnóstico de enfermagem segundo a classificação da NANDA, para a sistematização da assistência de enfermagem em aleitamento materno. Rev Latino-am Enfermagem 1997;5(2): 49-59.

13. Da Silva VM, De Oliveira Lopes MV, De Araújo TL, Ciol MA, De Carvalho EC. Clinical indicators of ineffective airway clearance in children with congenital heart disease. I Clin Nurs 2009; 18(5): 729-36.

14. Simpson T, Ivey I. Neonate with cardiac issues. Pediatric Nurs 2006; 32(6): 566-7.

15. Gibbins S, Maddalena P, Yamada I, Stevens B. Testing the satisfaction and feasibility of a computer-based teaching module in the neonatal intensive care unit. Advan Neonatal Care 2007; 7( 1$)$ : 43-9.

16. Orozco LC, Pinilla E, Vargas C, Buitrago DA, Domínguez DB, Peñaranda LM, Reyes DM, Rueda I. Factores asociados con el diagnóstico de enfermería deterioro parental en madres hasta los 24 años de edad, durante el primer año de crianza. Colomb Med 2007; 38(4): 79-88.

17. Viera CS, Rossi LA. Os diagnósticos de enfermagem da taxonomia da NANDA em mulheres com o filho prematuro hospitalizado e o sistema conceitual de King. Rev Latino-am Enfermagem 2000; 8(6): 110-16.

18. Lopes MHBM, Vale IN, Barbosa M. Opinião dos alunos Quanto ao ensino dos Diagnósticos de Enfermagem segundo a taxonomia da NANDA. Rev Esc Enferm USP 1997;3 I (1): 80-8.

19. Armitage G. Nursing assessment and diagnosis of respiratory distress in infants by children's nurses. I Clin Nurs 1999;8(1): 22-30.

20. Galligan M. Proposed guidelines for skin-to-skin treatment of neonatal hypothermia. MCN - Am I Maternal Child Nurs 2006; 3 I (5): 298-304.

2 I. Gamarra ICA, Carvajal BV, Suárez MI. Neonato crítico. Bogotá: 
Accofaben; 1998.

22. AlbuQuerQue CC, Nóbrega MML, Fontes WD. Sistematização da assistência de enfermagem a um binômio mãe/lactentes utilizando a teoria das necessidades humanas básicas e a CIPE@ versão 1.0. Cienc Cuid Saúde 2008; 7(3): 392-8.

23. Mefford LC. A theory of health promotion for preterm infants based on Levine's conservation model of nursing. Nurs Sci Quarterly 2004; 17(3): 260-6.

24. Lopes GK, Rossetto EG, Belei RA, Capobiango ID, Matsuo T. Estudo epidemiológico das infecções neonatais no Hospital Universitário de Londrina, Estado do Paraná. Acta Sci Health Sci 2008;39(1): 55-63.

25. ABO Nacional. A boca sob terapia intensiva. [online]. [citado em 20 abril 2009]. Disponível em:http://www.odontoex clusive.com/index.php?Option=com_content\&view $=$ article\&id $=55 \&$ Itemid $=40$
26. Delgado SE, Halpern R. Amamentação de prematuros com menos de 1500 gramas: funcionamento motor-oral e apego. Pró-Fono Rev Atual Cien 2005; 17(2): 141-52.

27. Carmona EV, Lopes MH. Content validation of parental role conflict in the neonatal intensive care unit. Int I Nurs Terminol Classif 2006; 17(1): 3-9.

28. Vale IA, Souza SR, Carmona EV. Nursing diagnoses identified during parent group meetings in a neonatal intensive care unit. Int I Nurs Terminol Classif 2005; 16(3-4): 65-73.

29. Scochi CGS, Riul MIS, Garcia CFD, Barradas LS, Pileggi SO. Cuidado individualizado ao peeueno prematuro: o ambiente sensorial em unidade de terapia intensiva neonatal. Acta Paul Enferm 200I; 14(1): 9-16.

30. Fonseca LMM, Leite AM, Mello DF de, Dalri MCB. Scochi CCS. Semiotécnica e semiologia do recém-nascido pré-termo: avaliação de um software educacional. Acta Paul Enferm 2008; 21: 543-8. 